\title{
Challenges and opportunities in social neuroscience
}

\author{
John T. Cacioppo and Jean Decety \\ Center for Cognitive and Social Neuroscience, University of Chicago
}

\begin{abstract}
Social species are so characterized because they form organizations that extend beyond the individual. The goal of social neuroscience is to investigate the biological mechanisms that underlie these social structures, processes, and behavior and the influences between social and neural structures and processes. Such an endeavor is challenging because it necessitates the integration of multiple levels. Mapping across systems and levels (from genome to social groups and cultures) requires interdisciplinary expertise, comparative studies, innovative methods, and integrative conceptual analysis. Examples of how social neuroscience is contributing to our understanding of the functions of the brain and nervous system are described, and societal implications of social neuroscience are considered.
\end{abstract}

\section{Keywords}

Social neuroscience; functional magnetic resonance imaging (fMRI); animal models; neuroimaging; lesion studies; affective and cognitive neuroscience

The past two decades have witnessed a remarkable rise in the number of investigations published under the rubric of social neuroscience. The discoveries conveyed by the titles of many of these reports (e.g., the neural basis of love, altruism, morality, generosity, trust) have piqued the interest of young investigators, funding agencies, the media, and laypeople alike. Such attention is a double-edged sword, however, as errors are exaggerated in importance, and oversimplifications create false expectations and, ultimately, disillusionment in what the field can contribute. A case in point is the accusation of voodoo correlations in neuroimaging studies of emotion, personality, and social cognition and the resulting controversy ${ }^{1-4}$. It is, of course, one thing to assume that neural processes underlie all psychological phenomenon, it is another to claim that a given brain region is the biological instantiation of complex psychological functions like the self, empathy or moral reasoning.

A decade ago, the field was characterized by some as defining social and cognitive functions and correlating these functions with regions of brain activation using functional neuroimaging, but the field has always had a broader foundation ${ }^{5}$. Moreover, social neuroscience involves manipulating and measuring phenomena at multiple levels of organization (i.e., at different scales of representation), and the emphasis is not simply on specifying associations across levels but on specifying the mechanisms responsible for these associations. Our purpose here is to examine developments and opportunities in the field of social neuroscience, with an emphasis on the breadth of disciplines, species, and methods

Address correspondence to the John Cacioppo at the Center for Cognitive and Social Neuroscience, University of Chicago, $5848 \mathrm{~S}$. University Avenue, Chicago, IL 60637. Cacioppo@uchicago.edu.

Conflicts of interest: None 
that it bridges. To explain this span, we begin by discussing what is unique about a social neuroscience perspective on neural structure and function.

\section{The perspective of social neuroscience}

Neuroscience refers to the collection of disciplines concerned with the structure and function of the nervous system and brain. The topic of study is so complex that it requires disparate basic, clinical, and applied disciplines to cover the terrain. Within neuroscience are crosscutting paradigms general perspectives that underlie a range of theories and methodologies in the field. The fulcrum for some of these perspectives rests squarely under constituent structures at different levels of organization, whereas the fulcrum for others falls under the function of the brain and nervous system. Illustrative of the latter is behavioral neuroscience, in which the nervous system and brain are viewed as instruments of sensation and response. Research representing this perspective tends to focus on topics such as learning, memory, motivation, homeostasis, sleep and biological rhythms, and reproduction and on the neural mechanisms underlying these behavioral functions. Cognitive neuroscience emerged as a distinct functional perspective in which the brain is viewed as an information processing organ, with a focus on topics such as attention, perception, representations, decision-making, memory systems, heuristics, reasoning, and executive functioning and on the neural mechanisms in the human brain that underlie these representations and processes ${ }^{6}$. Social neuroscience represents yet another broad perspective, one in which the emphasis is on the functions that are altered by or are derived from the association or interaction of conspecifics (imagined or real) and on the neural and hormonal mechanisms underlying these structures and functions.

Members of social species, by definition, create organizations beyond the individual. These superorganismal structures evolved hand in hand with psychological, neural, hormonal, cellular, and genetic mechanisms to support them because the consequent social behaviors helped these organisms survive, reproduce, and care for offspring sufficiently long that they too reproduced, thereby ensuring their genetic legacy. Social neuroscience seeks to specify the neural, hormonal, cellular, and genetic mechanisms underlying social behavior, and in so doing to understand the associations and influences between social and biological levels of organization.

As in behavioral neuroscience, animal models have been foundational to the field, as exemplified by the evidence marshaled by Cacioppo and Berntson ${ }^{7}$ nearly two decades ago for a doctrine of multi-level analyses in social neuroscience. Despite this early integration, contemporary social neuroscience investigations to elucidate the genetic, hormonal, cellular, and neural mechanisms of social behavior have grown from two largely separate root systems: one based on data from humans grounded in the discipline of psychology and the other based on data from animal models grounded in biology and biomedicine ${ }^{8,9}$. With a few notable exceptions in each group, there has been little communication between these two groups of scientists. In our view, this is a gap that must be filled for social neuroscience to reach its full potential. Not only is it important that those primarily interested in explaining human behavior begin to validate their hypotheses using animal models (lesions, pharmacology, etc), but it is also important for those primarily using animal models to understand the psychological constructs used by scientists who study mental and behavioral processes in humans to be better able to develop appropriate behavioral paradigms.

Moreover, interdisciplinary investigations of the commonalities and differences across social species (and across cultures within social species) in superorganismal structures, functions, and mechanisms are becoming increasingly important ${ }^{10-12}$. For instance, our understanding of social bonds was advanced when the concentration of oxytocin and 
vasopressin receptors were found in dopamine-rich areas of the brain in the monogamous prairie vole, whereas the concentration of these receptors were found located at sites distal to regions associated with reward and reinforcement in the more solitary montane and meadow voles 13,14 . Related work further suggests that polymorphisms in oxytocin receptors are involved in the sociality of voles ${ }^{15}$. birds ${ }^{16}$. and humans ${ }^{17}$. The animal research, in turn, has led to experimental studies in humans contrasting the effects of intranasal administration of oxytocin versus placebo ${ }^{18}$.

Humans are a somewhat unique social species in that our social institutions, civilizations, and cultures are highly developed, our territorial reach knows few boundaries, and our selection of and impact on the environment in which we live and the impact of this environment on genes is unmatched by any other species ${ }^{19,20}$. Some branches of social neuroscience, but particularly those that rely on neuroimaging, have focused on humans with an emphasis on the neural mechanisms involved in people thinking about the traits, mental states, and behavioral predispositions of others and have found a network of brain regions consistently active during such mentalizing, including the medial prefrontal cortex and the posterior part of the superior temporal sulcus ${ }^{21}$.

Our biology has helped shape the social environments we have created, and our social environment has helped shape our genes, gene expressions, brains, and bodies ${ }^{22,23}$. But we also share a biological heritage with other animals. Nonconscious influences are responsible for complex social behaviors in most if not all nonhuman social species. Nonconscious mechanisms have been conserved in humans, perhaps to a greater extent than is apparent from analyses of social cognition and behavior grounded in data from self-reports. Studies of other social species, animal models of specific aspects of human functioning, and investigations of our evolutionary lineage provide important information regarding these conserved mechanisms, and by doing so stimulates new theories and research on what might be exceptional to humans and what function our consciousness serves.

There is strong evidence, for instance, for regarding empathy as having deep evolutionary, neurological, and biochemical underpinnings. Even the most advanced forms of empathy in humans are build on more basic forms and remain connected to core mechanisms associated with affective communication, social attachment, parental care, and motivation to help. Although empathy has traditionally been examined as a unitary construct using behavioral methods, recent work in social and developmental neuroscience has begun to shed light on the neural circuitry that instantiate it ${ }^{24}$. This has been possible and productive because the construct of empathy has been broken down into component processes ${ }^{25,26}$. including bottom-up affective arousal, emotion understanding, motivation, and top-down emotion regulation. Further it is important to distinguish between empathy and sympathy (feelings of concern about the welfare of others). Although empathy and sympathy are often conflated, the two can be dissociated, and in humans although sympathy may stem from the apprehension of another's emotional state, it does not have to be congruent with the affective state of the other. Conceptual analyses such as this are crucial in designing and conducting interdisciplinary research that can bridge levels of organization within and across species in investigations of the evolutionary origins and ontogeny of empathy.

Converging research from animal behavior ${ }^{27}$, functional imaging studies in normal individuals, and lesion studies in neurological patients ${ }^{28}$ shows that empathy draws on a large array of brain structures and systems extending beyond the cortex (amygdala, insula, anterior cingulate cortex and orbitofrontal cortex), and also involve the autonomic nervous system (ANS), hypothalamic-pituitary-adrenal axis (HPA), and endocrine systems that regulate bodily states, emotion and reactivity (see Figure 1). Caring for others (sympathy) draws on general mammalian neural systems of reward, social attachment, and aversion ${ }^{29}$. 
Similarly, empathy is not unique to humans as many of the biological mechanisms are shared with other mammalian species ${ }^{30}$. In recent years, a number of studies have clearly reported the implication of oxytocin in human social cognition and empathy. In particular, its application via nasal spray reduces responses to social stress ${ }^{31}$. Intranasal administration of oxytocin, compared to placebo, reduces amygdala activation and modulates its coupling with brainstem regions that are involved in automatic fear reactivity ${ }^{32}$. Another study showed that a single dose of intranasally administrated oxytocin is sufficient to cause a substantial increase in the ability in affective mind reading on a test relying on the detection of subtle affective facial expression ${ }^{33}$. There is evidence that a naturally occurring genetic variation of the oxytocin receptor relates to both empathy and stress profiles. This was discovered in a study which tested how a polymorphism (rs53576) of the oxytocin receptor relates to empathy and stress reactivity ${ }^{34}$. Compared with individuals homozygous for the $G$ allele of rs53576 (GG), individuals with one or two copies of the A allele (AG/AA) exhibited lower behavioral and dispositional empathy. Furthermore, AA/AG individuals displayed higher physiological and dispositional stress reactivity than GG individuals, as determined by heart rate response during a startle anticipation task and an affective reactivity scale.

All these results concur with animal research suggesting a critical role for oxytocin in prosocial approach behavior and reactivity to social stress. It has also been proposed that the interaction between oxytocin and dopamine enhances the reward of social encounters, promoting the motivation to engage in social interactions ${ }^{35}$. Humans may be exceptional in the sense that high-level cognitive abilities such as executive function, language, and theory of mind are layered on top of phylogenetically older social and emotional capacities. These evolutionarily newer aspects of information processing expand the range and flexibility of the behaviors that can be driven by empathy like caring for and helping outgroup members or even individuals from different species ${ }^{26}$. These higher levels, however, operate on previous levels of organization and should not be seen as independent of, or conflicting with one another.

The focus of studying subcomponents of empathy can also be particularly useful from a developmental perspective, when it is the case that only some components of or precursors to more complex behaviors are observable. In addition, developmental studies can provide unique opportunities to see how the components of the system interact in ways not possible in adults, where all the components are fully mature and operational ${ }^{36}$. For example, a recent study used functional MRI to characterize developmental changes in brain activation in the neural circuits underpinning empathy and sympathy ${ }^{37}$. Fifty-seven individuals, aged from 7 to 40 years old, were presented with short animated visual stimuli depicting painful and non-painful situations involving either a person whose pain was accidentally caused or a person whose pain was intentionally inflicted by another individual. The stimuli were extensively validated prior the MRI scanning with eye-tracking and pupillary measures, as well as perceived intentionality and empathic concern judgments. Monotonic age-related changes in the amygdala, supplementary motor area, and posterior insula were found when participants were exposed to painful situations that were accidentally caused. When participants observed painful situations intentionally inflicted by another individual, agerelated changes were detected in the dorsolateral prefrontal and ventromedial prefrontal cortex, with a gradual shift in that latter region from its medial to its lateral portion. This pattern of activation reflects a change from a visceral emotional response critical for the analysis of the affective significance of stimuli to a more evaluative function. Furthermore, these functional changes supports the general notion that the development of affective processing from childhood to adulthood is accompanied by reduced activity within limbic affect processing systems, and increased involvement of other prefrontal systems ${ }^{38}$. 
In sum, social neuroscience can be viewed as a distinct paradigm in which to investigate the structure and function of the brain and nervous system, and to illuminate where we as a species fit within a broader biological context. Such an endeavor is challenging because it necessitates the integration of multiple levels and the explication of the mechanisms that link phenomena across these levels. Mapping across systems and levels (from genome to social groups and cultures) requires interdisciplinary expertise, comparative studies, innovative methods, and integrative conceptual analyses. To address many of the big questions in the field, therefore, requires larger and more interdisciplinary teams than were common in the prior century a trend that characterizes not only work in social neuroscience but in neuroscience ${ }^{39}$ and science more generally ${ }^{40,41}$.

\section{Multi-level analyses are critical to social neuroscience}

Human behavior can be parsed into levels of organization, ranging from the atomic to the geopolitical. What constitutes a level of organization, at least at the lower levels of scales of representation, is often guided by knowledge of anatomy and physiology, but the ultimate criterion is the usefulness of the posited organization in shedding light on some designated behavioral phenomenon. The terms "social" and "neuroscience" refer to sets of levels of organization, and their use is entirely compatible with monism. The constructs developed by behavioral and social scientists, for instance, provide a means of understanding highly complex activity without needing to specify each individual action by its simplest components, thereby providing a cognitively efficient way of referring to components of complex systems. These theoretical terms can help us understand brain function and, in turn, can be informed and refined through integration with theories and methods from the neurosciences. This is so because theories provide hypotheses about the manifestation of behavior across levels of organization, and the empirical tests can be used to support, reject, or refine these theories 42 .

The principle of multiple determinism specifies that a target behavior at one level of organization can have multiple antecedents within or across levels of organization. On the biological level, for instance, researchers identified the contribution of individual differences in the endogenous opioid receptor system in drug use, whereas on the social level investigators have noted the important role of social context. Both operate, and our understanding of drug abuse is incomplete if either level is excluded, as the social context has been found to interact with individual differences at the level of receptors in the brain ${ }^{43}$. Similarly, immune functions were once considered to reflect specific and nonspecific physiological responses to pathogens or tissue damage. It is now clear that immune responses are influenced by central nervous processes that, in turn, are affected by social interactions ${ }^{44}$. An understanding of human immunocompetence in everyday life will be inadequate in the absence of considerations of social and behavioral factors. An implication of this principle is that comprehensive theories of behavior will require a consideration of factors from multiple levels of organization. Accordingly, advances in the neurosciences and the social sciences may occur by increasing the scope of analysis to include the contributions of factors and processes from both social and neuroscientific perspectives.

An important corollary to this principle is that the mapping between elements across levels of organization becomes more complex (e.g., many-to-many) as the number of intervening levels of organization increases. One implication is that the likelihood of complex and potentially obscure mappings increases as one skips levels of organizations. This is perhaps one reason that going from the genotype to endophenotypes and from endophenotypes to phenotypes has proven to be more tractable than going directly from the genotype to phenotype ${ }^{45}$. 
The second principle is of nonadditive determinism, which specifies that properties of the whole are not always readily predictable by the simple sum of the properties of the parts. For instance, cortisol activation of the glucocorticoid receptor (GR) exerts broad antiinflammatory effects by inhibiting NF- $\mathrm{kB} /$ Rel transcription factors and other proinflammatory signaling pathways (e.g., JAK/STAT and IRF signaling) ${ }^{46,47}$. However, increased cortisol levels in socially isolated individuals are paradoxical in light of the fact that many isolation-linked diseases are driven by increased inflammation ${ }^{48-50}$. Given the broad anti-inflammatory effects of glucocorticoids, subjectively socially isolated people with elevated cortisol levels should be relatively protected from inflammation-mediated disease rather than having the increased risk empirically observed.

The mechanism responsible for this unexpected association is explicable when one takes into account the effects of perceived social isolation on biological processes that can render cells insensitive to the anti-inflammatory effects of glucocorticoids in vitro, such as decreased expression of the GR NR3Cl gene, post-translational modification of GR protein, increased expression of GR antagonists, and decreased activity of GR transcription cofactors ${ }^{51,52}$. Cole et al. ${ }^{53}$, for instance, analyzed genome-wide transcriptional activity in people who chronically experienced high versus low levels of perceived social isolation to assess alterations in the activity of transcription control pathways that might contribute to increased adverse health outcomes in social isolates. Promoter-based bioinformatic analyses showed under-expression of genes bearing anti-inflammatory glucocorticoid response elements and over-expression of genes bearing response elements for pro-inflammatory NF$\mathrm{\kappa B} /$ Rel transcription factors in individuals high, in contrast to low, in perceived social isolation. This reciprocal shift in pro- and anti-inflammatory signaling was not attributable to differences in circulating cortisol levels, or to other demographic, psychological, or medical characteristics. These data provide evidence that an individual's chronic connection or isolation vis-à-vis the social environment can alter gene transcriptional activity in a manner that accounts for the paradoxical association between cortisol and inflammation. The importance of these studies here derives from their demonstration of how the orderly mapping between the biological and social levels of organization would remain opaque until the analysis is extended across these levels of organization.

The third principle is of reciprocal determinism, which specifies that there can be mutual influences between biological and social factors in determining behavior. For example, Hackman et al. ${ }^{54}$ review evidence that socioeconomic status has effects on cognition and academic achievement in part through its effects on childhood brain development such as specialization for language in the left hemisphere (e.g., activation of the left, relative to right, inferior frontal gyrus during language tasks). Among the mediators of this "top down" effect are prenatal factors, parental care, and cognitive stimulation. Differences in neurodevelopment, in turn, affect executive functioning, academic achievement, and subsequent socioeconomic status. As illustrated in this research, the principle of reciprocal determinism implies that comprehensive accounts of behavior cannot be achieved if the biological or the social level of organization is considered unnecessary or irrelevant.

In sum, there is growing evidence that multilevel analyses spanning neural and social levels of organization can foster comprehensive accounts of cognition, emotion, behavior, and health. This is in part because the social environment shapes neural structures and processes and vice versa.

\section{Challenges of complexity}

Most of the complex functional constructs that are used in social neuroscience such as selfregulation, prejudice, attachment, empathy, and trust, can hardly be seen as natural kind 
sited somewhere in the brain and/or mapping onto an underlying biological mechanism in a one-to-one fashion. Instead, these constructs need to be broken down into sub-components with the challenge of avoiding narrow definitions that fail to capture the breadth of a function and vague concepts that may well resonate with lay knowledge and common sense but have little if any scientific validity and utility. Our review of research on empathy above is a case in point. Research on the "self" is a second case in point.

The question of the representation of the self in the brain has been the target of an impressive number of neuroscientific investigations during the past decade, as well ${ }^{55,56}$. The self is, itself, a complex construct and has included manifold dimensions including: the ecological self, the present self, the distant self, the experiential self, prereflexive self, mental self, core self, minimal self, spatial self, emotional self, autobiographical self, and the narrative self. An equally impressive number of neuroimaging studies and brain regions have been associated with the self including the medial prefrontal cortex, ventro-and dorsolateral prefrontal cortex, lateral parietal cortex, bilateral temporal poles, insula, and subcortical regions such as the brain stem, colliculi, and periaqueductal gray ${ }^{57}$. Such an inflation and heterogeneity of concepts reflects the challenge of bridging the phenomenological level of description with its neural underpinnings. It may be futile to seek a correlation between the self and brain processing without seriously breaking down the concept of self into component processes that have a logical validity and a functional (e.g., behavioral) anchor. And even then, it should not be assumed that there is a one-toone mapping between a component process and a neural structure.

In recent years, several neuroimaging studies have suggested that the neural basis of the selfreferential process is special and is uniquely mediated by a restricted area in the medial prefrontal cortex ${ }^{58-60}$. It remains controversial whether activity of this aspect of the medial prefrontal cortex (and other related brain regions) appears only during self-referential processes, however ${ }^{61}$. Based on a critical evaluation of the behavioral and neuroscience literature, Gillihan and Farah ${ }^{62}$ concluded that many of the claims for the special status of self-related processing are premature given the evidence and that the various self-related research programs do not seem to be illuminating a unitary, common system, despite individuals' subjective experience of a unified self ${ }^{63}$.

Scientific theories in social neuroscience seek to provide a mechanistic explanation for behavioral or brain function in terms of antecedents, structure, processes, and consequences. There are various forms of explanation that might be sought. For instance, one might seek to understand complex behavioral processes in terms of molecular or neural processes. Investigations seeking this form of explanation have the potential to contribute a great deal to our understanding of brain function. Alternatively, one might seek a mechanistic account in terms of psychological (e.g., cognitive, social, affective) constructs, information processing components, or computations underlying a behavioral phenomenon. Evidence from the neurosciences are perhaps not necessary in such studies, but it may prove useful as a source of hypotheses about what these constructs, components, or computations might be, or as a means of performing a crucial test between theories, made possible by breaking down the component processes of the psychological construct of interest and showing how, based on the prior literature on the brain, different predictions about what circuits should be activated can be derived from two or more theories ${ }^{64}$. As noted above, it is less useful to take a complex psychological construct and simply correlate it with regions in the brain to report neural correlates.

Despite the excitement about neuroimaging studies, it is clear that these methods are not sufficient to unravel the neurobiological mechanisms mediating social cognition and social behavior. It is equally clear that, because neuroimaging is noninvasive, it has an important 
role to play in the development, testing, and refinement of theories of complex psychological processes that are difficult to study in nonhuman animals.

Sound social neuroscience research and theory needs to take into account with equal importance data from three converging approaches: First, behavioral analyses and assessments of complex psychological constructs are foundational ${ }^{42,65}$. Psychological constructs need to be decomposed into component structures, representations, and processes that could plausibly be implemented by the brain. These, in turn, can be decomposed into the computations that are likely to be implemented by the complex neural machinery that constitutes the brain. What these might be will change with advances in theory, methods, and evidence. Tasks can then be defined that permit the isolation of one or more specific components, as verified by behavioral analyses, which in turn permit fine-grain analyses of brain-behavior associations. Work is still needed, as well, to determine how the neural components might be combined to produce distinct psychological processes. One metaphor is the Lego set, in which the computations performed in localized neural regions are fixed, but different pieces and configurations of these building blocks produce different psychological processes. An alternative metaphor is the periodic table in chemistry in which different neural component processes may have properties and affinities whose function (computation) depends on the network of areas with which they are combined. There is no evidence at present to favor either perspective, but the important point here is that they suggest there are very different ways of thinking about neural activity and psychological function that need yet to be sorted out.

Second, correlative evidence from the normal waking brain using a variety of measurement techniques provides important information. The brain does not operate exclusively at the spatial level of molecules, cells, nuclei, regions, circuits, or systems, nor does it operate exclusively at the temporal level of milliseconds, seconds, minutes, hours, or days.

Neuroimaging provides a partial view of brain activity within a very limited range of spatial and temporal levels. Importantly, the smallest regions detectable by fMRI still involve hundreds of thousands of cells, cells that are seldom homogeneous in their function or connections, so such studies identify neural circuitry only in a gross sense. For instance, different cells within the amygdala have been identified that respond to appetitive versus aversive stimuli ${ }^{66}$. Detection of amygdala activation by fMRI in response to appetitive and aversive stimuli does not mean the same neural circuitry has been activated. Therefore, converging measures that gauge neural events at different temporal and spatial scales are needed to provide a more complete picture of brain function.

Third, experimental evidence from animals and humans is critical. Neuroimaging is a correlative measure, so experimental studies including lesion, transcranial magnetic stimulation, and pharmacological interventions (e.g., ligands, drugs) in human and nonhuman animal are essential to further elucidate the causal role of any given neural structure, circuit, or process in a given task. Lesion method remains a fundamental and indispensable approach. Lesion is a means to test at the system level, the necessity and specificity of a particular region, can thus provide complementary information to neuroimaging data.

Each of these angles has limitations, but the confluence of the three can facilitate advances in our understanding of brain function. The point here is that neuroimaging represents an important part but only a part of the armamentarium that is needed, with the resulting knowledge more likely to be beneficial if it is combined with: (i) conceptual analyses that decompose complex psychological constructs into component structures, representations, processes, and computations; (ii) converging measures that gauge neural events at different temporal and spatial scales; (iii) behavioral measures that permit fine-grain analyses of 
brain-behavior associations; and (iv) experimental and nonhuman animal studies that test the putative role of specific brain structures, circuits, or processes at finer grain levels of organization.

In sum, neuroimaging work is leading to a rethinking of the parcelation of psychological and neural functions. The presence of so many open questions in the field is both a daunting challenge and an exciting opportunity. In the recent past, neuroimaging studies have been criticized for sounding like neo-phrenology. This issue has been exacerbated by the reliance on a simple version of the subtraction method wherein the identified brain region was equated with the psychological operation that differentiated the conditions. Such an approach can now be complemented by a consideration of how these regions operate within a broader set of brain regions that are involved. Functional connectivity analyses, studies of the interaction of brain networks in vivo using electrophysiological methods, and sophisticated causal modeling analyses in human and animal studies are permitting scientists to move beyond these criticisms ${ }^{67}$. Conceptual developments also have a role to play, as replacements and refinements in the lexicon of constructs are developed ${ }^{68}$. By combining multiple methods, advances can be expected in what constitutes elemental component processes (functional elements), the neural mechanisms (structural elements) underlying these functional elements, and the functional properties of interrelated networks of structural elements.

\section{Beyond the Laboratory}

Social neuroscience is an exciting and growing field of research, which clearly has the potential of many significant applications in various aspect of our life beyond the academia, including education, health and public policy. New ethical issues are arising as social neuroscience gives us unprecedented ways to understand the human mind and to predict, influence, and even control it ${ }^{69,70}$. One important illustration that has attracted attention from the criminal justice and intelligence communities is the study of the neural correlates of deception. Finding a reliable and invariant neural signature for lying has been a quest for millennia, and the use of MRI scanner has reactivated the debate over the sensitivity and reliability of such measures. Recent work on the neuroscience of deception raises many important questions, including whether deception processes are special in any way, or whether they rely on a set of general-purpose processes ${ }^{71,72}$. It also raises new questions about whether, when, and how to ensure the privacy of one's own mind.

Another example of the impact of neuroscience on the moral, legal, and policy issues relates to our understanding of the experience of agency (i.e., the awareness of being in control both of one's own actions and through them of events in the external world). Society requires that people are responsible for their actions and the consequences of those actions, and the notion of volition has been an important determinant in judgments of whether a person is guilty of criminal charges ${ }^{73}$. Recent work in philosophy, psychology, psychiatry, and neuroscience have called into question the validity of the notion of free will in human actions ${ }^{74}$. For instance, the prediction of decisions based on brain functions observed prior to the decision have led to suggestions that people may not have as much responsibility for their actions as assumed by the law. If one's conscious deliberations and decisions are predetermined by antecedent brain functions, the notion is that these deliberations and decisions are epiphenomenal. Even if this were true in the case of an isolated individual, these deliberations and decisions lead to actions toward and communications with others. The individual's interaction with another person leads to responses and a corresponding change in the initial person's brain function that is not entirely predictable based on his or her antecedent brain functions. The initial person's articulations and behavior in the interaction affects the second person's consciousness and underlying brain state, which in turn affects 
the initial person's consciousness and underlying brain states. The brain states of one can influence the brain states of others by what is expressed verbally, nonverbally, or behaviorally. Therefore, language and other deliberate actions can serve as important mediators between these brain states. This does not mean people necessarily have free will, but it does imply that consciousness (though nothing more than a brain function) may play a functional role in one's (subsequent) brain states through the effects of other's. As the neuroscience of decision making, agency and impulse control begins to offer a more detailed and specific account of the neurobiological processes leading to irresponsible or criminal behavior, neuroethics and law may not be able to ignore the expanding knowledge. Our intuitive understanding of people and what drive their behavior will be challenged.

To dramatize the distinction a bit, if cognitive neuroscience can be thought of as viewing the brain as a solitary computer, social neuroscience can be thought of as viewing the brain as a mobile, broadband computing device designed to connect to and work with other computing devices. Both perspectives are correct, of course, but each leads to somewhat different questions, approaches, insights, and perspectives. To understand the brain from the perspective of social neuroscience, it is crucial that we not settle simply for measurements across different levels of organization, we must also seek to delineate the mechanisms through which the observed processes operate and the more general principles that explain the operation of these mechanisms. The specification and tests of such mechanisms and principles will be advanced by the integration of animal and human models to provide a more comprehensive understanding of the complexity inherent to social processes, otherwise it will give a false and naïve view of how social phenomenon are implemented in biological mechanisms and how these processes can be investigated scientifically. Consequently, we believe that the validity of the concepts/constructs in social neuroscience and the generativity of the theories in the field will be advanced by embracing and building on the interdisciplinary nature of the field.

\section{Acknowledgments}

Preparation of this manuscript was supported by National Institutes of Health Grant No. RO1 AG034052-01.

\section{References}

1. Lieberman MD, Berkman ET, Wager TD. Correlations in Social Neuroscience Aren't Voodoo: Commentary on Vul et al. (2009). Perspectives on Psychological Science. 2009; 4:299-307.

2. Nichols T. Commentary on Vul et al (2009). Puzzlingly High Correlations in fMRI Studies of Emotion, Personality, and Social Cognition. Perspectives on Psychological Science. 2009; 4:291293.

3. Vul E, et al. Puzzlingly High Correlations in fMRI Studies of Emotion, Personality, and Social Cognition. Perspectives on Psychological Science. 2009; 4:274-290.

4. Vul E, et al. Reply to Comments on Puzzlingly High Correlations in fMRI Studies of Emotion, Personality, and Social Cognition. Perspectives on Psychological Science. 2009; 4:319-324.

5. Cacioppo, JT., et al. Foundations in Social Neuroscience. Vol. 49. MIT Press; Cambridge Mass: 2002.

6. Gazzaniga, MS. The cognitive neurosciences. MIT Press; Cambridge Mass: 1995. <http://www.worldcat.org/title/cognitive-neurosciences/oclc/29318436\&referer=brief_results>

7. Cacioppo JT, Berntson GG. Social psychological contributions to the decade of the brain. Doctrine of multilevel analysis. The American psychologist. 1992; 47:1019-28. [PubMed: 1510329]

8. Cacioppo JT, Bertson GG, Decety J. A brief history of social neuroscience. Handbook of the History of Social Psychology. 2011

9. Matusall S, Kaufmann IM, Christen M. The emergence of social neuroscience as an academic discipline. Handbook of Social Neuroscience. 2011 
10. Blumstein DT. Towards an integrative understanding of social behavior: new models and new opportunities. Frontiers in Behavioral Neuroscience. 2010; 4:1-9. [PubMed: 20126432]

11. Byrne RW, Bates L. a Primate social cognition: uniquely primate, uniquely social, or just unique? Neuron. 2010; 65:815-30. [PubMed: 20346757]

12. Norman GJ, et al. Selective influences of oxytocin on the evaluative processing of social stimuli. Journal of psychopharmacology (Oxford, England). 2010:1-14.10.1177/0269881110367452

13. Lim MM, et al. Enhanced partner preference in a promiscuous species by manipulating the expression of a single gene. Nature. 2004; 429:754-757. [PubMed: 15201909]

14. Young LJ, Wang Z. The neurobiology of pair bonding. Nature neuroscience. 2004; 7:1048-54.

15. Hammock, EaD; Young, LJ. Functional microsatellite polymorphism associated with divergent social structure in vole species. Molecular biology and evolution. 2004; 21:1057-63. [PubMed: 15014156]

16. Goodson JL, et al. Mesotocin and nonapeptide receptors promote estrildid flocking behavior. Science (New York, NY). 2009; 325:862-6.

17. Lucht MJ, et al. Associations between the oxytocin receptor gene (OXTR) and affect, loneliness and intelligence in normal subjects. Progress in neuro-psychopharmacology \& biological psychiatry. 2009; 33:860-6. [PubMed: 19376182]

18. Campbell A. Oxytocin and human social behavior. Personality and social psychology review : an official journal of the Society for Personality and Social Psychology, Inc. 2010; 14:281-95.

19. Bell AV, Richerson PJ, McElreath R. Culture rather than genes provides greater scope for the evolution of large-scale human prosociality. Proceedings of the National Academy of Sciences of the United States of America. 2009; 106:17671-4. [PubMed: 19822753]

20. Gazzaniga, M. Human : the science behind what makes us unique. Ecco; New York: 2008. at <http://www.worldcat.org/title/human-the-science-behind-what-makes-us-unique/oclc/ 179807190\&referer=brief_results>

21. Baron-Cohen, S. Theory of mind. Psychology Press; Hove: 2007. at <http://www.worldcat.org/title/theory-of-mind/oclc/488257120\&referer=brief_results>

22. Chiao JY, Blizinsky KD. Culture-gene coevolution of individualism-collectivism and the serotonin transporter gene. Proceedings Biological sciences / The Royal Society. 2010; 277:529-37. [PubMed: 19864286]

23. Hackman, Da; Farah, MJ.; Meaney, MJ. Socioeconomic status and the brain: mechanistic insights from human and animal research. Nature Reviews Neuroscience. 2010; 11:651-659.

24. Decety, J.; Ickes, W. The social neuroscience of empathy. MIT Press; Cambridge Mass: 2009. at $<$ http://www.worldcat.org/title/social-neuroscience-of-empathy/oclc/ 243845315\&referer=brief_results $>$

25. Decety J, Jackson PL. The functional architecture of human empathy. Behavioral and cognitive neuroscience reviews. 2004; 3:71-100. [PubMed: 15537986]

26. Decety J. Dissecting the neural mechanisms mediating empathy. Emotion Review. 2011 in press.

27. Langford DJ, et al. Social modulation of pain as evidence for empathy in mice. Science (New York, NY). 2006; 312:1967-70.

28. Hornak J, et al. Changes in emotion after circumscribed surgical lesions of the orbitofrontal and cingulate cortices. Brain : a journal of neurology. 2003; 126:1691-712. [PubMed: 12805109]

29. Moll J, et al. Human fronto-mesolimbic networks guide decisions about charitable donation. Proceedings of the National Academy of Sciences of the United States of America. 2006; 103:15623-8. [PubMed: 17030808]

30. Parr LA, Waller B. The evolution of human emotion. Evolution of the Nervous System. 2007:447472.

31. Heinrichs M, et al. Social support and oxytocin interact to suppress cortisol and subjective responses to psychosocial stress. Biological Psychiatry. 2003; 54:1389-1398. [PubMed: 14675803]

32. Kirsch P, et al. Oxytocin modulates neural circuitry for social cognition and fear in humans. The Journal of neuroscience : the official journal of the Society for Neuroscience. 2005; 25:11489-93. [PubMed: 16339042] 
33. Domes G, et al. Oxytocin attenuates amygdala responses to emotional faces regardless of valence. Biological psychiatry. 2007; 62:1187-90. [PubMed: 17617382]

34. Rodrigues SM, et al. Oxytocin receptor genetic variation relates to empathy and stress reactivity in humans. Proceedings of the National Academy of Sciences of the United States of America. 2009; 106:21437-41. [PubMed: 19934046]

35. Insel TR, Young LJ. The neurobiology of attachment. Nature reviews Neuroscience. 2001; 2:12936.

36. De Haan, M.; Gunnar, MR. The brain in a social environment. Why study development?. Handbook of Developmental Social Neuroscience. 2009. at <http://www.worldcat.org/title/handbook-of-developmental-social-neuroscience/oclc/ 299126301\&referer=brief_results>

37. Decety J, Michalska KJ. Neurodevelopmental changes in the circuits underlying empathy and sympathy from childhood to adulthood. Developmental Science. 2010 in press. 10.1111/j.14677687.2009.00940.x

38. Killgore WDS, Yurgelun-Todd D. a Unconscious processing of facial affect in children and adolescents. Social neuroscience. 2007; 2:28-47. [PubMed: 18633805]

39. Silver R, et al. Neurotech for neuroscience: unifying concepts, organizing principles, and emerging tools. The Journal of neuroscience : the official journal of the Society for Neuroscience. 2007; 27:11807-19. [PubMed: 17978017]

40. Jones BF, Wuchty S, Uzzi B. Multi-university research teams: Shifting impact, geography, and stratification in science. Science. 2008; 433:1259-1262. [PubMed: 18845711]

41. Wuchty S, Jones BF, Uzzi B. The increasing dominance of teams in production of knowledge. Science (New York, NY). 2007; 316:1036-9.

42. Cacioppo JT, Decety J. What Are the Brain Mechanisms on Which Psychological Processes Are Based? Perspectives on Psychological Science. 2009; 4:10-18.

43. Dalley JW, et al. Nucleus accumbens D2/3 receptors predict trait impulsivity and cocaine reinforcement. Science (New York, NY). 2007; 315:1267-70.

44. Hawkley, LC., et al. Loneliness, dysphoria, stress and immunity: A role for cytokines. 2007.

45. Cacioppo JT, Berntson GG, Thisted RA. Multilevel investigations: Conceptual mappings and perspectives. Biosocial Surveys. 2008:367-380.

46. Rhen T, Cidlowski J. a Antiinflammatory action of glucocorticoids--new mechanisms for old drugs. The New England journal of medicine. 2005; 353:1711-23. [PubMed: 16236742]

47. Collado-Hidalgo A, et al. Inflammatory biomarkers for persistent fatigue in breast cancer survivors. Clinical cancer research : an official journal of the American Association for Cancer Research. 2006; 12:2759-66. [PubMed: 16675568]

48. Levy JA. Pathogenesis of human immunodeficiency virus infection. Microbiological reviews. 1993; 57:183-289. [PubMed: 8464405]

49. Hansson GK. Inflammation, atherosclerosis, and coronary artery disease. The New England journal of medicine. 2005; 353:429-30. author reply 429-30.

50. Coussens LM, Werb Z. Inflammation and cancer. Nature. 2002; 420:860-7. [PubMed: 12490959]

51. Pace TWW, Hu F, Miller AH. Cytokine-effects on glucocorticoid receptor function: relevance to glucocorticoid resistance and the pathophysiology and treatment of major depression. Brain, behavior, and immunity. 2007; 21:9-19.

52. Bamberger CM, Schulte HM, Chrousos GP. Molecular Determinants of Glucocorticoid Receptor Function and Tissue Sensitivity to Glucocorticoids. Endocrine Reviews. 1996; 17:245-261. [PubMed: 8771358]

53. Cole SW, et al. Social regulation of gene expression in human leukocytes. Genome biology. 2007; 8:R189. [PubMed: 17854483]

54. Hackman D, Farah MJ, Meaney MJ. Socioeconomic status and the brain: mechanistic insights from human and animal research. NATURE REVIEWS NEUROSCIENCE. 2010; 11:651-659.

55. Decety J, Sommerville JA. Shared representations between self and other: a social cognitive neuroscience view. Trends in Cognitive Sciences. 2003; 7:527-533. [PubMed: 14643368] 
56. Klein SB, Gangi CE. The multiplicity of self: Neuropsychological evidence and its implications for the self as a construct in psychological research. The Year in Cognitive Neuroscience 2010: New York Academy of Sciences. 2010; 1191:1-15.

57. Northoff G, et al. Self-referential processing in our brain--a meta-analysis of imaging studies on the self. NeuroImage. 2006; 31:440-57. [PubMed: 16466680]

58. D'Argembeau A, et al. The neural basis of personal goal processing when envisioning future events. Journal of cognitive neuroscience. 2010; 22:1701-13. [PubMed: 19642887]

59. Kelley WM, et al. Finding the self? An event-related fMRI study. Journal of cognitive neuroscience. 2002; 14:785-94. [PubMed: 12167262]

60. Mitchell JP, Banaji MR, Macrae CN. The link between social cognition and self-referential thought in the medial prefrontal cortex. Journal of cognitive neuroscience. 2005; 17:1306-15. [PubMed: 16197685]

61. Yaoi K, Osaka N, Osaka M. Is the self special in the dorsomedial prefrontal cortex? An fMRI study. Social neuroscience. 2009; 4:455-63. [PubMed: 19588282]

62. Gillihan SJ, Farah MJ. Is self special? A critical review of evidence from experimental psychology and cognitive neuroscience. Psychological bulletin. 2005; 131:76-97. [PubMed: 15631554]

63. Legrand D, Ruby P. What is self-specific? Theoretical investigation and critical review of neuroimaging results. Psychological review. 2009; 116:252-82. [PubMed: 19159156]

64. Waytz A, et al. Making sense by making sentient: Effectance motivation increases anthropomorphism. Journal of personality and social psychology. 2010; 99:410-435. [PubMed: 20649365]

65. Posner, M.; Raishle, ME. Images of mind. Scientific American Library; New York: 1994. at <http://www.worldcat.org/title/images-of-mind/oclc/29598229\&referer=brief_results>

66. Paton JJ, et al. The primate amygdala represents the positive and negative value of visual stimuli during learning. Nature. 2006; 439:865-70. [PubMed: 16482160]

67. Ullsperger, M.; Debener, S. Simultaneous EEG and fMRI : recording, analysis, and application. Oxford University Press; New York: 2010. at

<http://www.worldcat.org/title/simultaneous-eeg-and-fmri-recording-analysis-and-application/ oclc/428770799\&referer=brief_results>

68. Poldrack RA. Mapping mental function to brain structure: How can cognitive neuroimaging succeed? Perspectives on Psychological Sciencei. in press.

69. Farah, M. Neuroethics: an introduction with readings. MIT Press; Cambridge Mass: 2010. at <http://www.worldcat.org/title/neuroethics-an-introduction-with-readings/oclc/ 498365634\&referer=brief_results>

70. Farah MJ. Ethical, legal and societal issues in social neuroscience. The Handbook of Social Neuroscience. 2011

71. Ganis, G. Neural correlates of deception : a special issue of social neuroscience. Psychology; Hove: 2009. at $<$ http://www.worldcat.org/title/neural-correlates-of-deception-a-special-issue-of-socialneuroscience/oclc/436265514\&referer=brief_results>

72. Monteleone GT, et al. Detection of deception using fMRI: better than chance, but well below perfection. Social neuroscience. 2009; 4:528-38. [PubMed: 18633832]

73. Haggard P, Tsakiris M. The experience of agency: Feelings, judgments, and responsibility. Current Directions in Psychological Science. 2009; 18:242-246.

74. Kircher, T.; David, AS. The self in neuroscience and psychiatry. Cambridge University Press; Cambridge UK ;;New York: 2003. at $<$ http://www.worldcat.org/title/self-in-neuroscience-and-psychiatry/oclc/ 50554454\&referer=brief_results> 


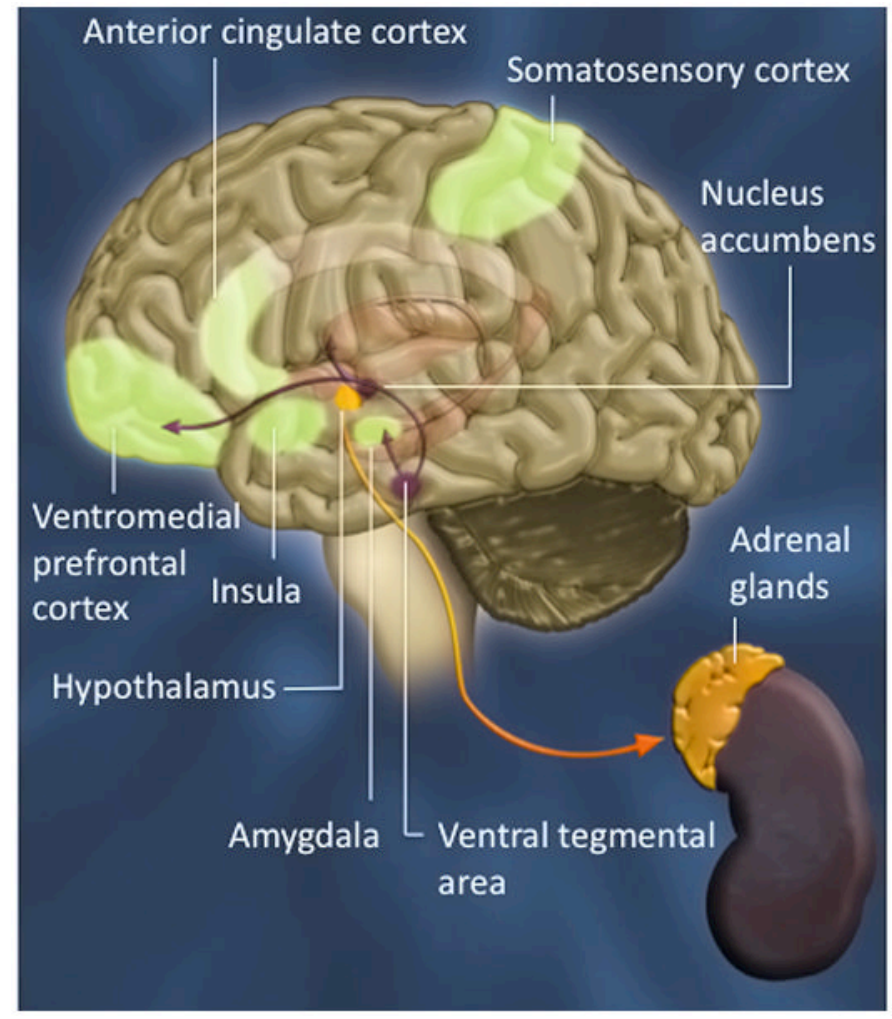

Figure 1.

Empathy is implemented by a complex network of distributed, often recursively connected, interacting neural regions, as well as autonomic and neuroendocrine processes implicated in social behaviors and emotional states. 


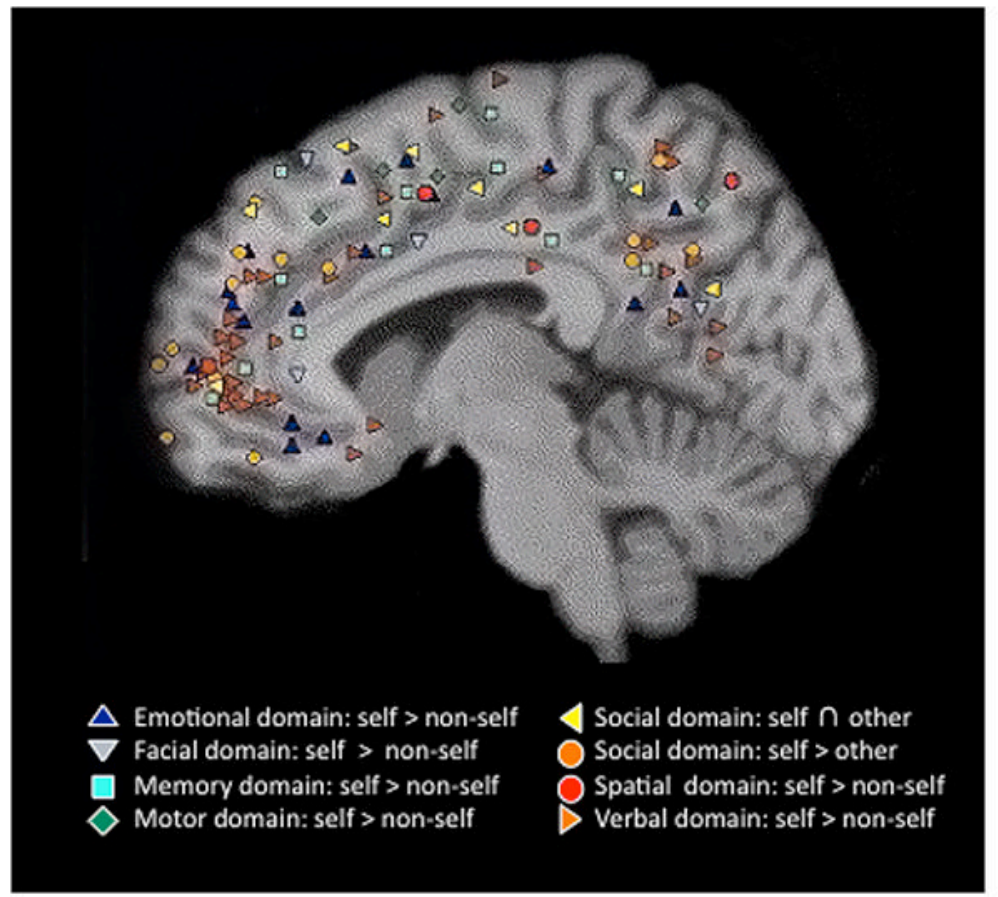

Figure 2.

Outcome of a meta-analysis of activation foci reported in $27 \mathrm{fMRI}$ studies published between 2000 and 2004. These studies investigate brain activity during self-related tasks in different self-related domains. Reproduced with permission from Northoff et al. (2006). 